Supplementary Information

\title{
Stress or strain does not impact sorption in stiff mesoporous materials
}

\author{
M. Bossert, A. Grosman, I. Trimaille, ${ }^{\dagger}$ C. Noûs, ${ }^{\ddagger}$ and E. Rolley*, \\ † Sorbonne Université, CNRS, Institut des NanoSciences de Paris, INSP, F-75005 Paris, \\ France \\ † Laboratoire Cogitamus $13 / 4$ rue Descartes, 75005 Paris, France \\ ฯ Laboratoire de Physique de l'Ecole Normale Supérieure, ENS, Université PSL, CNRS, \\ Sorbonne Université, Université de Paris, F-75005 Paris, France \\ E-mail: rolley@phys.ens.fr, ORCID0000-0003-1333-2541
}

\section{Contents}

White Light Interferometry

Relation between the optical thickness $L$ and the amount of adsorbed fluid $N$ S3

Number of page: 4

Total number of figure: 3 


\section{White Light Interferometry}

Principle - WLI is routinely used to measure thin optical films. The sample is illuminated with white light, using a tungsten lamp and a bundle of optical fibers. The reflected light is collected by a second fiber and analyzed with a high resolution spectrometer (Ocean Optics HR4000) as shown in figure S1a.

The incoming beam is reflected at both the interfaces of the porous layer (see Fig. S1b), due to the difference in optical indexes $n_{v}, n_{e f f}$ and $n_{b}$ of vapor, porous silicon and bulk silicon respectively. As a result of the interference between the two beams, the spectrum of the reflected light displays oscillations whose period depends on the optical thickness $L$ of the porous layer (Fig. S2). The optical thickness $L=2 n_{e f f} l_{\|}$depends on the effective index $n_{\text {eff }}$ and on the thickness $l_{\|}$of the porous layer.

Obtaining the optical thickness - The reflected intensity $I$ can be written as $I=$ $A \cos \left(4 \pi n_{e f f} l_{\|} / \lambda\right)+B$, where $A$ and $B$ are slowly varying functions of the wavelength $\lambda$, as they depend on the 3 indexes and on the absorption in the porous layer. In term of the wave number $\sigma \equiv 1 / \lambda, I(\sigma)=A \cos (2 \pi L \sigma)+B$. Thus $L$ can be very easily obtained by performing a Fourier transform of $I(\sigma)$, as it has been done in previous works where WLI is used to detect or measure adsorption. ${ }^{1-3}$

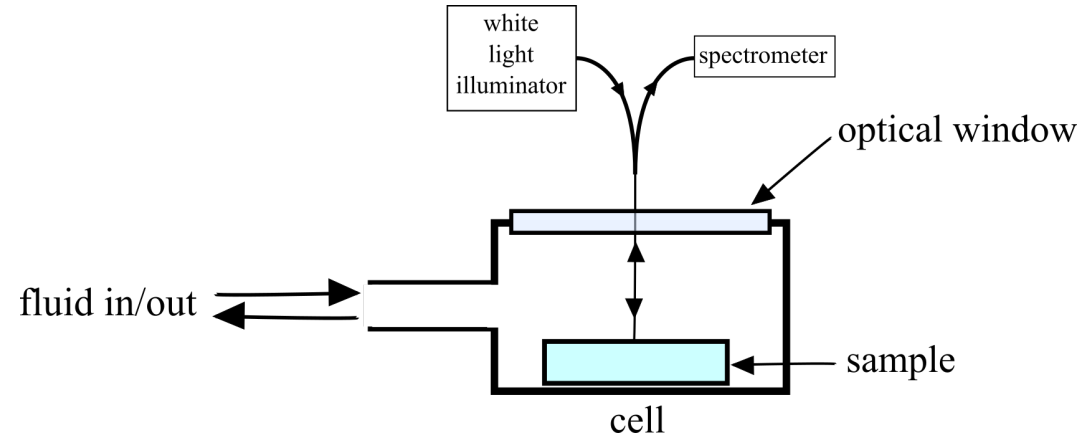

(a)

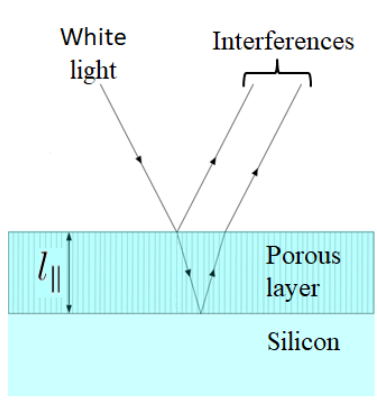

(b)

Figure S1: (a) Schematic of the setup. (b) Principle of White Light Interferometry for a supported porous layer.

As the pressure $P$ in the cell increases, fluid adsorbs in the porous layer. Hence, the 
effective optical index $n_{\text {eff }}$ increases, and so does the optical thickness of the layer and therefore the frequency of the oscillations in the intensity $I$. This is easily visible in Fig. S2b, where the relative change in $L$ between the two curves is about $4 \%$. In Fig. S2a, the relative change in $L$ is much smaller, about $0.2 \%$ : the change in the period of the oscillations is not visible. However, as a consequence of the large interference order $L / \lambda$, the shift of the spectrum, that is the change of the phase, is easily detected. Systematic tests have revealed that, in our case, the phase measurement is more precise than the period measurement, so that we have chosen this method for obtaining the isotherms $\Delta L$ vs $P$ shown in the article. The absolute determination of $L$ still requires the measure of the period of the oscillations. Note also that a single spectrum is enough to obtain $L$ through a FFT of the signal, but the unwrapping of the phase requires continuous acquisition.

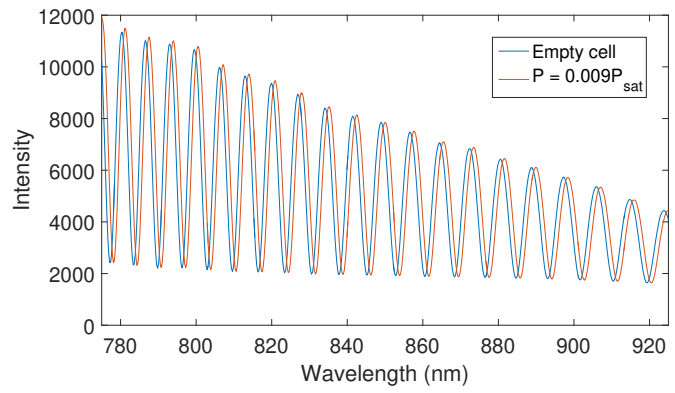

(a)

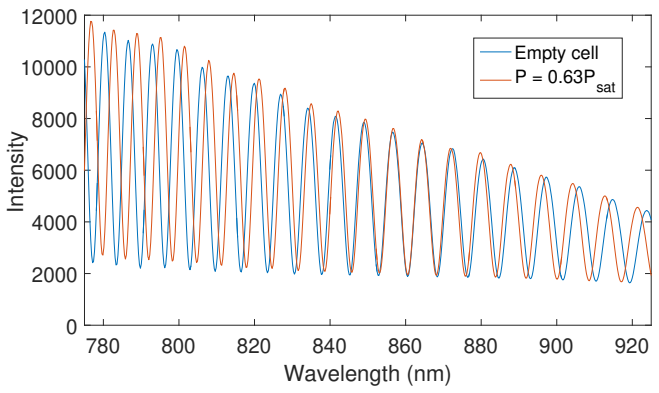

(b)

Figure S2: Optical spectrums for a supported porous layer (70\% porosity and $20 \mu \mathrm{m}$ ) at zero pressure (blue curve) and with hexane at pressure $P$ in the cell (red curve). For this sample $L(P=0)=95 \mu \mathrm{m}$ and $\Delta L_{M}=13.36 \mu \mathrm{m}$.

(a) $P=0.009 P_{\text {sat }}, \Delta L=0.18 \mu \mathrm{m}$, (b) $P=0.63 P_{\text {sat }}, \Delta L=3.79 \mu \mathrm{m}$

\section{Relation between the optical thickness $L$ and the amount}

\section{of adsorbed fluid $N$}

Figure S3 displays the two nitrogen isotherms $N(P)$ and $\Delta L=L(P)-L(0)$ that we have measure in order to check whether the optical thickness varies linearly with $N$ (see Ap- 
pendix).

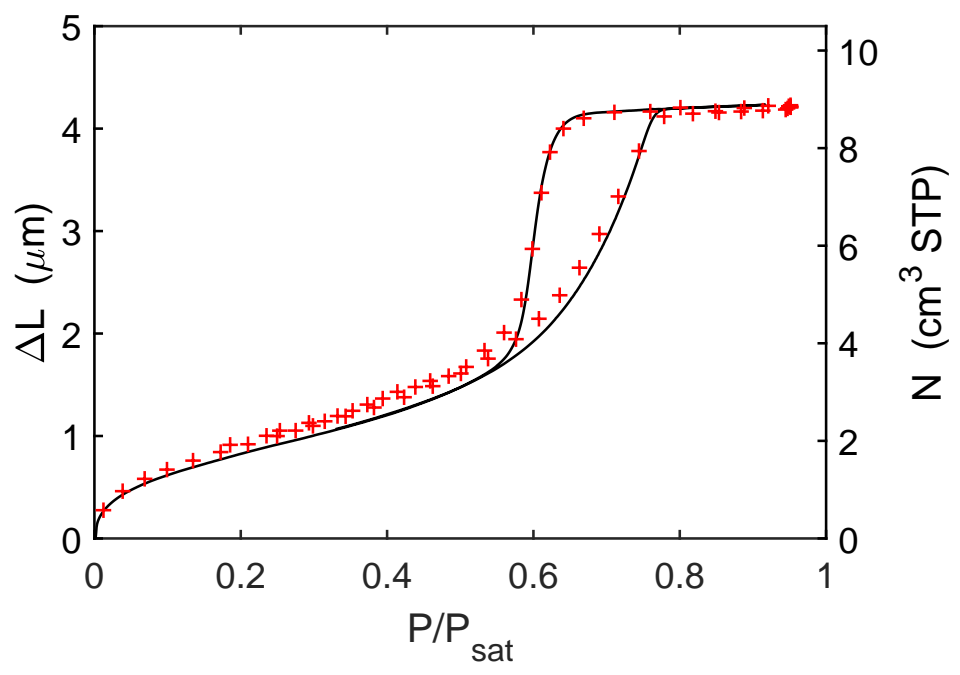

Figure S3: Optical and volumetric isotherms for a 50\% porosity sample with $20 \mu \mathrm{m}$ pores (fluid: nitrogen, $\mathrm{T}=77.4 \mathrm{~K}, L(0)=107 \mu \mathrm{m})$.

\section{References}

(1) Pacholski, C.; Sartor, M.; Sailor, M. J.; Cunin, F.; Miskelly, G. M. Biosensing using porous silicon double-layer interferometers: reflective interferometric Fourier transform spectroscopy. Journal of the American Chemical Society 2005, 127, 11636-11645.

(2) Casanova, F.; Chiang, C. E.; Li, C.-P.; Roshchin, I. V.; Ruminski, A. M.; Sailor, M. J.; Schuller, I. K. Gas adsorption and capillary condensation in nanoporous alumina films. Nanotechnology 2008, 19, 315709.

(3) Casanova, F.; Chiang, C. E.; Ruminski, A. M.; Sailor, M. J.; Schuller, I. K. Controlling the role of nanopore morphology in capillary condensation. Langmuir 2012, 28, 68326838. 